\title{
CompLement C5 Antibodies for decreasing brain injury after aneurysmal Subarachnoid Haemorrhage (CLASH): study protocol for a randomised controlled phase II clinical trial
}

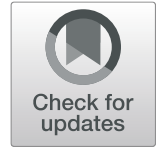

Inez Koopman* (D, Gabriel J. E. Rinkel, and Mervyn D. I. Vergouwen on behalf of the CLASH study group

\begin{abstract}
Background: The inflammatory response after aneurysmal subarachnoid haemorrhage (aSAH) has been associated with early brain injury, delayed cerebral ischaemia, poor functional outcome, and case fatality. In experimental SAH studies, complement C5 antibodies administered shortly after SAH reduced brain injury with approximately $40 \%$. Complement component C5 may be a new therapeutic target to reduce brain injury and hereby improve the outcome after aSAH. We aim to investigate the pharmacodynamic efficacy and safety of eculizumab (complement C5 antibody) in patients with aSAH.

Methods: A randomised, controlled, open-label, phase II clinical trial with blinded outcome assessment. Eculizumab (1200 mg) is administered intravenously $<12 \mathrm{~h}$, on day 3 and on day 7 after ictus. Patients in the intervention group receive prophylactic antibiotics for 4 weeks, and those with a central line or an external ventricular shunt and a positive fungal or yeast culture also receive prophylactic antifungal therapy for 4 weeks. The primary outcome is C5a concentration in the cerebrospinal fluid (CSF) on day 3 after ictus. Secondary outcomes include the occurrence of adverse events, inflammatory parameters in the blood and CSF, cerebral infarction on magnetic resonance imaging, and clinical and cognitive outcomes. We aim to evaluate 26 patients with CSF assessments, 13 in the intervention group and 13 in the comparator group. To compensate for early case fatality and inability to obtain CSF, we will include 20 patients per group.
\end{abstract}

Discussion: The CLASH trial is the first trial to investigate the pharmacodynamic efficacy and safety of eculizumab in the early phase after aSAH.

Trial registration: Netherlands Trial Register NTR6752. Registered on 27 October 2017

European Clinical Trials Database (EudraCT) 2017-004307-51

Keywords: Subarachnoid haemorrhage, Eculizumab, Efficacy, Proof-of-concept, Safety, Complement, Inflammation

* Correspondence: i.koopman-2@umcutrecht.nl

Department of Neurology and Neurosurgery, UMC Utrecht Brain Centre, University Medical Centre Utrecht, Utrecht University, Bolognalaan 2-48, 3584

CJ Utrecht, the Netherlands

(c) The Author(s). 2020 Open Access This article is licensed under a Creative Commons Attribution 4.0 International License, which permits use, sharing, adaptation, distribution and reproduction in any medium or format, as long as you give appropriate credit to the original author(s) and the source, provide a link to the Creative Commons licence, and indicate if changes were made. The images or other third party material in this article are included in the article's Creative Commons licence, unless indicated otherwise in a credit line to the material. If material is not included in the article's Creative Commons licence and your intended use is not permitted by statutory regulation or exceeds the permitted use, you will need to obtain permission directly from the copyright holder. To view a copy of this licence, visit http://creativecommons.org/licenses/by/4.0/. The Creative Commons Public Domain Dedication waiver (http://creativecommons.org/publicdomain/zero/1.0/) applies to the data made available in this article, unless otherwise stated in a credit line to the data. 


\section{Administrative information}

Note: the numbers in curly brackets in this protocol refer to the SPIRIT checklist item numbers. The order of the items has been modified to group similar items (see http://www.equator-network.org/reporting-guidelines/spirit-2013-statement-defining-standard-protocolitems-for-clinical-trials/).

\begin{tabular}{|c|c|}
\hline Title $\{1\}$ & $\begin{array}{l}\text { CompLement C5 Antibodies for } \\
\text { decreasing brain injury after aneurysmal } \\
\text { Subarachnoid Haemorrhage (CLASH): } \\
\text { study protocol for a randomised } \\
\text { controlled phase II clinical trial }\end{array}$ \\
\hline Trial registration $\{2 a$ and $2 b\}$. & $\begin{array}{l}\text { Netherlands Trial Register: NTR6752, } \\
\text { https://www.trialregister.n//trial/6579. } \\
\text { Registered on } 27 \text { October } 2017 . \\
\text { European Clinical Trials Database: } \\
\text { EudraCT 2017-004307-51 } \\
\text { See Additional File } 1 \text { for all items in the } \\
\text { WHO trial registration data set for this } \\
\text { study. }\end{array}$ \\
\hline Protocol version $\{3\}$ & 08-07-2020, version 11.0 \\
\hline Funding $\{4\}$ & $\begin{array}{l}\text { Netherlands Organization for Health } \\
\text { Research and Development, the Dutch } \\
\text { Brain Foundation, and Alexion } \\
\text { Pharmaceuticals. }\end{array}$ \\
\hline Author details $\{5 \mathrm{a}\}$ & $\begin{array}{l}\text { Department of Neurology and } \\
\text { Neurosurgery, UMC Utrecht Brain } \\
\text { Centre, Matthias van Geuns building, } \\
\text { room 02.15, University Medical Centre } \\
\text { Utrecht, Utrecht University, Bolognalaan } \\
\text { 2-48, 3584 CJ Utrecht, the Netherlands } \\
\text { Tel: +31-88-7571441. Email: i.koopman-2 } \\
\text { @umcutrecht.nl }\end{array}$ \\
\hline $\begin{array}{l}\text { Name and contact } \\
\text { information for the trial } \\
\text { sponsor }\{5 b\}\end{array}$ & $\begin{array}{l}\text { University Medical Centre Utrecht } \\
\text { Heidelberglaan } 100 \\
3584 \text { CX Utrecht } \\
\text { Tel: +3188 } 7555555\end{array}$ \\
\hline Role of sponsor $\{5 c\}$ & Monitoring and management. \\
\hline
\end{tabular}

\section{Introduction}

\section{Background and rationale $\{6 a\}$}

Early brain injury and delayed cerebral ischaemia are important determinants of poor outcome after aneurysmal subarachnoid haemorrhage (aSAH) [1]. No treatment exists to reduce early brain injury, and the effects of current strategies to prevent delayed cerebral ischaemia are only modest [2]. The inflammatory response is considered to play a key role in the pathogenesis of early brain injury and delayed cerebral ischaemia after aSAH [3-6]. Previous studies found that the complement cascade is activated in patients with $\mathrm{SAH}$ and associated with poor functional outcome [7, 8]. Complement components $\mathrm{C} 3 \mathrm{a}$ and $\mathrm{C} 5 \mathrm{a}$ are proinflammatory anaphylatoxins that can induce vasoconstriction and activate coagulation [9-11], processes that have been implicated in the pathophysiology of early brain injury and delayed cerebral ischaemia [1, 12]. C5specific antibodies, which prevent the formation of C5a, have been shown to reduce microglia activation and cell death by $40 \%$ in an SAH mouse model [13]. C5 antibodies (eculizumab) are already used for other inflammatory diseases such as neuromyelitis optica and myasthenia gravis $[14,15]$. The aim of this trial is to investigate the pharmacodynamic efficacy (proofof-concept) and safety of eculizumab in patients with aSAH. As this is the first trial to investigate the use of eculizumab in aSAH patients, the effective dosing regimen for aSAH patients is unknown. In our previous study, we found that the $\mathrm{C} 5 \mathrm{a}$ concentration in the cerebrospinal fluid (CSF) of aSAH patients is highly increased (> 1400 times) compared to the C5a concentration in CSF from patients with unruptured intracranial aneurysms [13]. We therefore decided upon administration of a high dose of eculizumab $(1200 \mathrm{mg})$ with repeated drug administration to prevent a wash-out effect.

\section{Objectives $\{7\}$}

The aim of this trial is to investigate the pharmacodynamic efficacy (proof-of-concept) and safety of eculizumab in patients with aSAH.

\section{Trial design $\{8\}$}

The CLASH trial is a randomised, controlled, openlabel, phase II clinical trial with blinded outcome assessment (PROBE) to assess the pharmacodynamic efficacy and safety of eculizumab in patients with aSAH.

\section{Methods: participants, interventions, and outcomes \\ Study setting $\{9\}$}

This study will be conducted at the University Medical Centre Utrecht (UMC Utrecht), a tertiary referral centre.

\section{Eligibility criteria $\{10\}$}

The inclusion and exclusion criteria are presented in Table 1.

\section{Who will take informed consent? $\{26 \mathrm{a}\}$}

The physician on call will contact the investigators if an eligible patient is admitted. Written informed consent to participate in the study will be asked from the patient or legally authorized representative after the diagnosis is discussed with the patient and/or the legally authorized representative. Consent is asked by the physician on call or the investigators. If the physician on call is too busy, one of the investigators will come to the hospital to ask for consent. The consent form includes information about the study's rationale, study procedures, and 
Table 1 Inclusion and exclusion criteria

Inclusion criteria
- SAH confirmed by CT and aneurysm by CTA or DSA
- Admission to the UMC Utrecht < $12 \mathrm{~h}$ after ictus
- Age 18 years and older
Exclusion criteria
- Life expectancy < 10 days
- Pregnant or breastfeeding women
- Participation in another clinical therapeutic study
- History of splenectomy or asplenia
- Haematologic malignancy
- Patients receiving chemotherapy
- Patients who will undergo or underwent an organ transplantation
- Patients with myasthenia gravis, glucose-6-phosphate dehydrogen-
ase (G6PD) deficiency, or tuberculosis
- Patients who are or will be treated by plasmapheresis or
haemodialysis
- Patient with a creatinine clearance of < 30 or serum creatinine levels
of $>169 \mu$ mol/l
- Patients with a known hereditary complement deficiency
- Patients allergic to eculizumab, proteins derived from mouse
products, or other monoclonal antibodies
- Patients allergic to (prophylactic) antibiotic treatment for Neisseria
meningitidis (quinolones or ceftriaxone)
- If on admission, it is likely that the aneurysm can only be treated
with extracranial-intracranial bypass surgery
- If based on head imaging, it will be unlikely that CSF can be
obtained at day 3 after ictus
- Patients with an ongoing infection on admission which is not
Patients who were treated $>4$ times with antibiotics during the last year
- PAmmunosuppressive therapy

SAH subarachnoid haemorrhage, CT computed tomography, CTA computed tomography angiography, DSA digital subtraction angiography, UMC Utrecht University Medical Centre Utrecht, CSF cerebrospinal fluid

possible benefits and risks. If it will not be possible to obtain consent and administer eculizumab $<12 \mathrm{~h}$ after ictus, the patient and/or legally authorized representative will not be approached for trial participation. The patient or representative will receive as much time as needed to decide about trial participation. However, it is noted during the conversation with the patient and/or legally authorized representative that trial participation can only take place if inclusion $<12 \mathrm{~h}$ after ictus is possible.

\section{Additional consent provisions for collection and use of participant data and biological specimens $\{26 \mathrm{~b}\}$}

Informed consent is asked for the collection and use of participant data and biological specimens. No ancillary studies will be conducted.

\section{Interventions}

Explanation for the choice of comparators $\{6 \mathrm{~b}\}$

The comparator group in our study is composed of patients with SAH who receive care as usual. No placebo treatment and no standard antibiotics or antifungal therapy is administered to the comparator group unless clinically indicated. Although we would have preferred to make use of a placebo comparator for eculizumab, it was not possible to manufacture placebo eculizumab, ciprofloxacin, and fluconazole infusions in our hospital within a reasonable time window and reasonable costs. In addition, because this trial is a phase II proof-of-concept trial with a primary outcome based on laboratory parameters, the absence of a placebo treatment in the comparator group will not affect our primary outcome.

\section{Intervention description $\{11$ a}

The intervention consists of intravenous infusion with eculizumab $1200 \mathrm{mg}$ at three different time points: < $12 \mathrm{~h}$, on day 3, and day 7 after ictus (Fig. 1). The day of ictus is defined as day 1. To decrease the risk of (meningococcal) infection due to eculizumab treatment, patients in the intervention group receive prophylactic treatment with ciprofloxacin during the first 4 weeks after ictus. During the recruitment phase, after the inclusion of the 6th patient, we changed our protocol based on a serious adverse event (SAE) that occurred (cerebral fungal infection in a patient with external ventricular shunt). After the amendment, patients in the intervention group with a central line or an external ventricular shunt and a positive fungal or yeast culture receive prophylactic ciprofloxacin and fluconazole for the first 4 weeks after ictus. Throat and rectal swabs are performed weekly in the intervention group during the inhospital stay to test for fungus or yeast carriership/ colonization and (multi-) drug resistance. In consultation with the microbiologist and infectious disease specialist, prophylactic treatment will be switched if (1) swabs are positive for microorganisms that require treatment and are not covered by our prophylactic regimen and (2) resistant microbial phenotypes are found.

\section{Criteria for discontinuing or modifying allocated interventions $\{11 \mathrm{~b}\}$}

Treatment with eculizumab will be discontinued in the following events:

- Anaphylactic shock after infusion of eculizumab. In case of a mild allergic reaction, the infusion rate will be slowed down. 


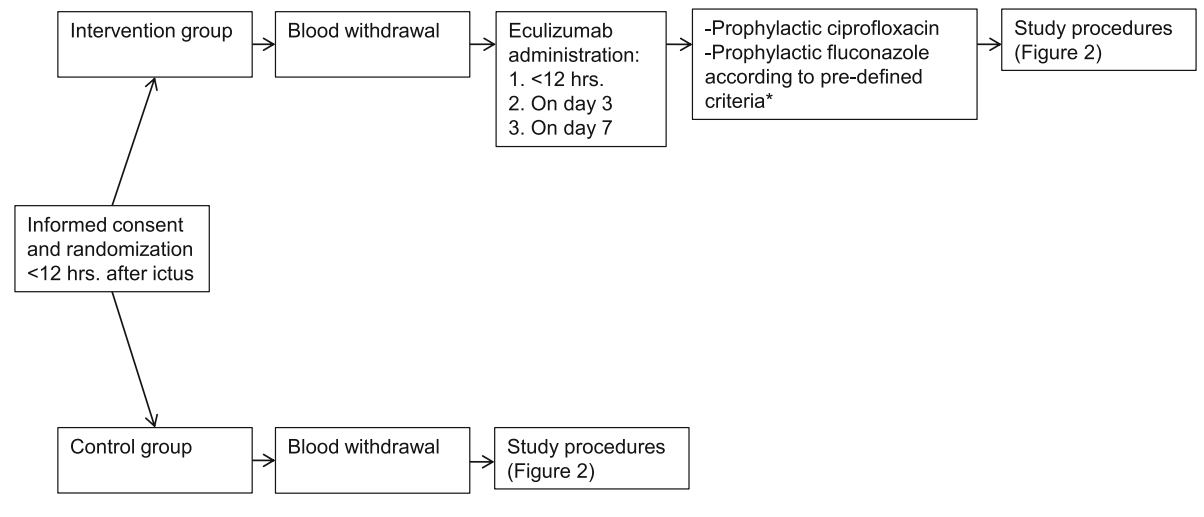

*Patients in the intervention group with a central line or an external ventricular shunt and a positive fungal or yeast culture will receive prophylactic fluconazole in addition to prophylactic ciprofloxacin.

Fig. 1 Treatment allocation.

hrs. $=$ hours

- Patients with Neisseria meningitidis meningitis, CSF culture-proven.

- Patients with Neisseria meningitidis sepsis, blood culture-proven.

- Other medical reasons for which the treating physician or investigator deems it necessary to discontinue treatment.

No modifications to the dosing schedule will be performed except when a mild allergic reaction occurs. If treatment with eculizumab is halted, other study procedures will continue according to the study protocol.

\section{Strategies to improve adherence to interventions $\{11 \mathrm{c}\}$} The investigator visits the trial participants regularly during their in-hospital stay to explain all study procedures, provided the clinical condition allows such an explanation. If the trial participant is discharged, the participant receives an explanation about the importance of adherence to the study protocol and a safety card to inform other physicians about trial participation. After discharge, trial participants are seen at the outpatient clinic by the same investigator, who then again explains all study procedures.

\section{Relevant concomitant care permitted or prohibited during the trial $\{11 d\}$}

All concomitant care or interventions are allowed except for the concomitant care listed in the exclusion criteria (e.g. immunosuppressive medication, chemotherapy).

\section{Provisions for post-trial care $\{30\}$}

Trial participants will be insured by the UMC Utrecht clinical trial participant insurance.

\section{Outcomes $\{12\}$}

The primary outcome is C5a concentration in CSF on day 3 after ictus. The difference in $\mathrm{C} 5 \mathrm{a}$ concentrations between the intervention and comparator groups will be assessed (either the difference in the mean or median concentrations, depending on the distribution of the data). CSF is obtained by either lumbar puncture or sampling from an external lumbar or ventricular drain. Secondary outcomes are listed in Table 2 . The mean or median scores between the intervention and comparator groups will be assessed at various time points (Fig. 2) for all secondary outcomes except for the World Federation of Neurosurgical Societies (WFNS) score and Modified Ranking Scale (mRS) score for which the proportion of patients with a specific score will be compared.

Table 2 Secondary outcomes

1. The occurrence of AEs and SAEs. Blinded assessment of infections will be performed by an expert panel consisting of a microbiologist and an infectious disease specialist.

2. Blood and CSF parameters of inflammation.

3. Eculizumab concentration in the blood and CSF.

4. Daily neurological condition measured by the GCS.

5. Neurological condition measured by the NIHSS and WFNS scores on day 14 after ictus. If the patient is discharged earlier, the NIHSS and WFNS scores will be performed before discharge.

6. Cerebral infarction defined as infarction identified on brain MRI after the exclusion of procedure-related infarctions [16].

7. Cognition measured by the MoCA.

8. Quality of life measured by the EQ-5D-5L questionnaire.

9. Functional outcome measured by the mRS score. Telephone interviews will be conducted by a qualified person who is blinded for allocation.

AEs adverse events, SAEs serious adverse events, CSF cerebrospinal fluid, GCS Glasgow Coma Score, NIHSS National Institutes of Health Stroke Scale, WFNS World Federation of Neurosurgical Societies, MRI magnetic resonance imaging, MoCA Montreal Cognitive Assessment, mRS Modified Ranking Scale 


\begin{tabular}{|c|c|c|c|c|c|c|c|c|c|c|c|c|c|c|}
\hline \multirow[b]{2}{*}{ Measurements } & \multicolumn{14}{|c|}{ Days after ictus $\rightarrow$} \\
\hline & 1 & 2 & 3 & 4 & 5 & 6 & 7 & 8 & 9 & 10 & 11 & 12 & 13 & 14 \\
\hline Blood withdrawal & $\Delta^{*}$ & $\Delta$ & & $\Delta$ & & $\Delta$ & & & $\Delta$ & & & $\Delta$ & & $\Delta$ \\
\hline CSF sample & & & $\boldsymbol{\Delta}^{\# !}$ & & & & & & & & & & & \\
\hline $\begin{array}{l}\text { Neurological } \\
\text { Examination (GCS) }\end{array}$ & $\Delta$ & $\Delta$ & $\Delta$ & $\Delta$ & $\Delta$ & $\Delta$ & $\Delta$ & $\Delta$ & $\Delta$ & $\Delta$ & $\Delta$ & $\Delta$ & $\Delta$ & $\Delta$ \\
\hline WFNS score & $\Delta$ & & & & & & & & & & & & & $\mathbf{\Delta}^{*}$ \\
\hline NIHSS score & & & & & & & & & & & & & & $\Delta^{*}$ \\
\hline Brain MRI & & & & & & & & & & & & & & $\Delta^{ \pm}$ \\
\hline $\begin{array}{l}\text { Questionnaire AEs } \\
\text { and SAEs }\end{array}$ & & & & & & & & & & & & & & \\
\hline MoCA & & & & & & & & & & & & & & \\
\hline EQ-5D-5L & & & & & & & & & & & & & & \\
\hline $\begin{array}{l}\text { Modified Rankin } \\
\text { Scale }\end{array}$ & & & & & & & & & & & & & & \\
\hline
\end{tabular}

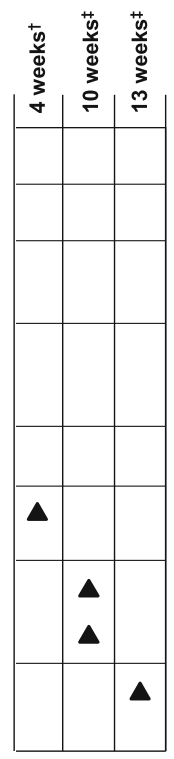

* Blood withdrawal will be performed before the first eculizumab administration; \# CSF will be obtained before the second eculizumab administration; $¥$ If the patient is discharged earlier, the NIHSS- and WFNS score will be performed before discharge; \pm If the patient is discharged earlier or clinically unstable, the MRI will be performed before discharge or postponed to a maximum of 28 days after ictus after which the MRI will be cancelled. If day 14 is during the weekend, the MRI will be performed before or after the weekend; $\dagger^{+}+$- one week $\ddagger+/$ - two weeks.

Fig. 2 Study procedures.

CSF, cerebrospinal fluid; GCS, Glasgow Coma Score; WFNS, World Federation of Neurosurgical Societies; NIHSS, National Institutes of Health Stroke Scale; MRI, magnetic resonance imaging; AEs, adverse events; SAEs, serious adverse events; MoCA, Montreal Cognitive Assessment; EQ-5D-5L, standardized instrument for use as a measure of health outcome

\section{Participant timeline $\{13\}$}

The time schedule of enrolment, intervention, assessments, and visits for participants can be found in Fig. 2.

\section{Sample size $\{14\}$}

The sample size calculation is based on a previous study with eculizumab in patients with neuromyelitis optica [14]. In that study, C5 concentration in CSF was measured in 11 patients before and after treatment with eculizumab. In six patients, C5 was undetectable after treatment was started, and in the remaining five patients, the mean C5 concentration in CSF decreased with 58\%. For the CLASH trial, we conservatively assumed an overall reduction of $\mathrm{C} 5$ concentration in CSF with $55 \%$ and extrapolated this to a similar reduction in C5a concentration in CSF. Based on $\alpha=$ 0.05 and $\beta=0.20$ and a standard deviation of $50 \%, 13$ patients are needed in each group. The group size will be increased to 20 patients per group, taking into account an assumed mortality rate of $25 \%$ and 2 patients per group who refuse a lumbar puncture in a later phase despite giving informed consent earlier.

\section{Recruitment $\{15\}$}

Eligible study participants are identified by the physician on call. Because consent for trial participation is asked after the diagnosis is discussed, the information about the diagnosis and the trial can be overwhelming for the patient and family. We developed a lay-men 3D medical animation explaining what a subarachnoid haemorrhage is. The medical animation is shown to the patient and/or family on an iPad and facilitates a conversation about trial participation. In addition, the Subarachnoid Haemorrhage Patient Association endorsed our clinical trial, and information about this trial was posted on their website.

\section{Assignment of interventions: allocation} Sequence generation $\{16 \mathrm{a}\}$

A computer-generated block randomization is used to randomise patients. No stratification factors were used.

\section{Concealment mechanism \{16b\}}

Not applicable, this trial is an open-label trial with blinded outcome assessment.

\section{Implementation $\{16 c\}$}

Patients are randomised by the investigators via a centralized secured website after informed consent from the patient or legally authorized representative is obtained. Assignment occurs computer-generated. Directly after randomization, the investigators receive an 
email with the allocation. Patients are allocated 1:1 to either (1) the intervention arm or (2) care as usual.

\section{Assignment of interventions: blinding Who will be blinded $\{17 \mathrm{a}\}$}

The use of coded samples will allow blinded measurement of C5a concentration by the laboratory technician. In addition, functional outcome measured by the mRS score will be conducted by a qualified person who is blinded for allocation. Blinded assessment of infections will be performed by an expert panel consisting of a microbiologist and an infectious disease specialist. Data analysts will not be blinded to allocation, because this trial is a proof-of-concept trial with a primary outcome based on laboratory parameters.

\section{Procedure for unblinding if needed $\{17 b\}$}

Not applicable.

\section{Plans for assessment and collection of outcomes \{18a\}}

Data collection is the same for the intervention and comparator groups (Fig. 2). Each patient is assigned a code upon randomization by a computer. Data are collected from the electronic patient system and then transferred to an electronic case report form (E-CRF) by the investigator under the code assigned upon randomization. If data are not available in the electronic patient system, a paper form is used after which the data are transferred to the E-CRF, also under the code assigned upon randomization. The electronic forms are a mix of pre-existing forms (e.g. GCS, NIHSS, mRS, and EQ-5D-5L) and forms designed by the investigator. Data quality checks are performed by a contract research organization according to the approved monitor plan ( $25 \%$ of all data entered will be checked). In addition, the E-CRF makes use of reference values where applicable. All data will be stored on a secured server.

\section{Plans to promote participant retention and complete follow-up $\{18 b\}$}

As specified in 11c, the participant receives a face-toface explanation about the importance of adherence to the protocol. In addition, participants will be asked if their phone number is valid. Study visits to the outpatient clinic are planned on the same day as the patient's standard of care appointment at the rehabilitation centre. Participants who choose to leave the trial are asked whether the collected data can be used for this research. If permission is provided, their data will be handled as data from other trial participants. If permission is not provided, their data will be deleted from the ECRF and the paper forms will be destroyed.

\section{Data management $\{19\}$}

The decryption key will be stored securely and is only accessible by the investigators. Data management procedures can be found in the data management plan provided as Additional File 2.

\section{Confidentiality $\{27\}$}

Data will be pseudonymized to guarantee confidentiality. Pseudonymized patient data will only be accessible by the investigators, the study monitor, and the health authorities, if required. The personal data collected and stored for the purpose of this study will be treated in accordance with the provisions of the General Data Protection Regulation (GPDR: Regulation EU 2016/679). Samples are de-identified and stored under UNI EN ISO 9001: 2015 regulations.

Plans for collection, laboratory evaluation, and storage of biological specimens for genetic or molecular analysis in this trial/future use $\{33\}$

The CSF and blood sampling, processing, storage, and immunoassays are described in Additional File 3.

\section{Statistical methods}

Statistical methods for primary and secondary outcomes \{20a\}

The primary analysis will be based on the per-protocol principle in which patients with CSF assessments will be included. Patients in the intervention group with CSF assessments are included if they received the first eculizumab infusion.

\section{Primary analysis}

Groups will be compared with an independent $t$ test or Mann-Whitney $U$ test, dependent on the distribution of data. If the proportion of patients categorized according to the Prognosis on Admission of Aneurysmal Subarachnoid Haemorrhage (PAASH) scale and the median Hijdra score differs between the intervention and comparator groups, a multivariable logistic regression analysis with adjustment for these variables will be performed.

\section{Secondary analyses}

Inflammatory parameters in the blood and daily Glasgow Coma Score (GSC) will be analysed with a linear mixed model. CSF inflammatory parameters in both groups will be compared by means of an independent $t$ test or Mann-Whitney $U$ test, dependent on the distribution of the data. To compare the NIHSS score, cognition, and quality of life, a chi-square or Fisher's exact test will be applied. A proportional odds model will be used to assess the effect of eculizumab on WFNS score and mRS score [17]. 
Interim analyses $\{21 b\}$

An interim analysis will be performed based on SAE reporting, outcome, or case fatality after the inclusion of 20 patients. The data safety monitoring board (DSMB) can advise early termination of the trial if there is evidence of severe harm based on SAE reporting, outcome, or case fatality.

Methods for additional analyses (e.g. subgroup analyses) $\{20 b\}$

Not applicable.

Methods in analysis to handle protocol non-adherence and any statistical methods to handle missing data $\{20 \mathrm{c}\}$ Missing data will not be imputed.

Plans to give access to the full protocol, participant-level data, and statistical code $\{31 \mathrm{c}\}$

The dataset of this study is not publicly available but is available from the corresponding author upon reasonable request.

\section{Oversight and monitoring}

Composition of the coordinating centre and trial steering committee $\{5 \mathrm{~d}\}$

This is a monocentre trial. The trial steering committee consists of multiple experts in the field of subarachnoid haemorrhage and infectious diseases and experts who have experience with treatment of patients with eculizumab. The trial steering committee provides guidance for general trial conduct.

Composition of the data monitoring committee, its role, and reporting structure $\{21 \mathrm{a}\}$

An independent data safety monitoring board (DSMB) will oversee the safety and overall conduct of the CLAS $\mathrm{H}$ trial. The DSMB consists of two neurologists and one clinical epidemiologist. The chair has previous experience in serving on DSMBs and experience in chairing meetings.

\section{Adverse event reporting and harms $\{22\}$}

Safety will be examined by ongoing monitoring of SAEs and suspected unexpected serious adverse reactions (SUSARs) and by an interim analysis. Listings of infections will be reported every 2 months to the DSMB chairman. The DSMB can advise early termination of the trial if there is evidence of severe harm based on SAE reporting, outcome, or case fatality. SAEs that can be expected based on the patient population include rebleeding, per-procedural aneurysm rupture, hydrocephalus, hyponatraemia, delayed cerebral ischaemia, nosocomial infections, Tersons's syndrome, epilepsy, and delirium. All AEs, SAEs, and SUSARs will be collected systematically by reviewing the electronic patient system and by reporting of the patient spontaneously or at 4 weeks after ictus. All adverse events will be reported in the supplementary material of the trial's publication.

Frequency and plans for auditing trial conduct $\{23\}$

A contract research organization will audit trial conduct following the approved monitor plan. This includes 2-3 visits a year in which the in- and exclusion criteria, informed consent forms, source data, and SAEs reporting forms are verified.

\section{Plans for communicating important protocol amendments to relevant parties (e.g. trial participants, ethical committees) $\{25\}$}

The coordinating investigator will submit amendments for review to the ethics committee of the UMC Utrecht. Upon approval, all parties involved will be informed about the amendment, and the protocol in the clinical trial registry will be updated.

\section{Dissemination plans $\{31 \mathrm{a}\}$}

After the clinical trial is completed, an article will be written for publication in an international journal. The results will also be presented at an international conference.

\section{Discussion}

In aSAH patients, an inflammatory response occurs in the subarachnoid space shortly after the bleeding. This inflammatory response has been associated with early brain injury, delayed cerebral ischaemia, poor functional outcome, and case fatality [1, 3-5]. In experimental SAH studies, treatment with complement C5 antibodies shortly after SAH reduced brain injury with approximately 40\% [13]. Complement component C5 may be an important target to reduce brain injury and hereby improve the outcome after aSAH. The CLASH trial is the first phase II trial to investigate the pharmacodynamic efficacy and safety of eculizumab in aSAH patients.

We chose an open-label design with blinded outcome assessment for several reasons: (1) we deemed it unethical to subject patients in the comparator group to 4 weeks of prophylactic treatment with ciprofloxacin and fluconazole, (2) it was not possible to manufacture placebo ciprofloxacin and fluconazole infusions at our hospital within a reasonable time window and reasonable costs, and (3) this trial is a proof-of-concept trial with a primary outcome based on laboratory parameters. We do not expect that ciprofloxacin or fluconazole will influence the C5a concentration in the CSF. However, the outcomes that are self-reported and assessed by 
clinicians not blinded to allocation can be biassed by the open-label design.

The CLASH trial is designed as a proof-of-concept trial and is not powered to assess the effectiveness of treatment with eculizumab. Safety is an important outcome of this trial. Eculizumab treatment increases the risk of infection. Central lines or external drains can provide a point of entry for microbes. aSAH patients will therefore be closely monitored during the in-hospital stay and receive prophylactic antibiotics, antifungal therapy if necessary, and a patient safety card with instructions. If our trial demonstrates the efficacy and safety of eculizumab in aSAH patients, the next step will be to plan a phase III trial.

\section{Trial status}

8 July 2019-version 11.0

The first patient has been recruited in October 2018. In September 2020, 20 patients have been enrolled. The recruitment is anticipated to be completed by 1 April 2021, with a follow-up period until 1 July 2021. Protocol modifications will be communicated to relevant parties. The results of this trial will be published in a peerreviewed scientific journal.

\section{Supplementary Information}

Supplementary information accompanies this paper at https://doi.org/10. 1186/s13063-020-04838-6.

Additional file 1. WHO trial registration data set.

Additional file 2. Data management plan.

Additional file 3. Sampling, processing, storage, and immunoassays.

\begin{abstract}
Abbreviations
aSAH: Aneurysmal subarachnoid haemorrhage; CLASH: CompLement C5 Antibodies for decreasing brain injury after aneurysmal Subarachnoid Haemorrhage; CSF: Cerebrospinal fluid; DSMB: Data safety monitoring board; E-CRF: Electronic case report form; GCS: Glasgow Coma Score; mRS: Modified Ranking Scale; PAASH: Prognosis on Admission of Aneurysmal Subarachnoid Haemorrhage; PROBE: Prospective Randomised Open Blinded End-point; SAE: Serious adverse event; SUSAR: Suspected unexpected serious adverse reaction; UMC Utrecht: University Medical Centre Utrecht; WFNS: World Federation of Neurosurgical Societies; ZonMw: Netherlands Organization for Health Research and Development
\end{abstract}

\section{Acknowledgements}

We would like to thank all UMC Utrecht neurology residents for their help with patient recruitment and B. Zweedijk for her contribution to the data collection.

CLASH study group:

I. Koopman ${ }^{1}$, R.W.P. Tack ${ }^{1}$, H.F. Wunderink ${ }^{2}$, A.H.W. Bruns ${ }^{3}$, I. van der Schaaf ${ }^{4}$, K.A. Gelderman ${ }^{5}$, J.P. Greving ${ }^{6}$, A.J.C. Slooter ${ }^{7}$, A. van der Zwan ${ }^{1}$, M. Bartels ${ }^{8}$, G.J.E. Rinkel', M.D.I. Vergouwen ${ }^{1}$

${ }^{1}$ Department of Neurology and Neurosurgery, UMC Utrecht Brain Centre, University Medical Centre Utrecht, Utrecht University, Utrecht, the Netherlands

${ }^{2}$ Department of Medical Microbiology, University Medical Centre Utrecht, Utrecht University, Utrecht, the Netherlands

${ }^{3}$ Department of Internal Medicine and Infectious Diseases, University Medical Centre Utrecht, Utrecht University, Utrecht, the Netherlands
${ }^{4}$ Department of Radiology, University Medical Centre Utrecht, Utrecht University, Utrecht, the Netherlands

${ }^{5}$ Sanquin Diagnostic Services, Amsterdam, the Netherlands

${ }^{6} J$ ulius Centre, University Medical Centre Utrecht, Utrecht University, Utrecht, the Netherlands

${ }^{7}$ Department of Intensive Care Medicine, UMC Utrecht Brain Centre,

University Medical Centre Utrecht, Utrecht University, Utrecht, the

Netherlands

${ }^{8}$ Department of Pediatric Hematology, University Medical Centre Utrecht, Utrecht University, Utrecht, the Netherlands

\section{Authors' contributions}

IK developed the research protocol, is responsible for conducting the study, and drafted the manuscript. GJER contributed to the study's design and revised the manuscript. MDIV conceptualized the study, is responsible for conducting the study, and provided input for the research protocol and manuscript. All authors read and approved the final manuscript. Authorship will be determined by role in study design, data collection, data analysis, and writing of the manuscript. We have no intended use of professional writers.

\section{Funding}

The study is funded by the Netherlands Organization for Health Research and Development (ZonMw), the Dutch Brain Foundation (PTO grant 95105015), and Alexion Pharmaceuticals (ISR grant 100237). ZonMw and the Dutch Brain Foundation are not involved in the trial design and data collection, analysis, and interpretation. The study drug is supplied by Alexion Pharmaceuticals, in addition to a small investigator-sponsored research grant. The dosage regimen for this study was decided in consultation with Alexion Pharmaceuticals. Alexion Pharmaceuticals is not involved in any other aspects of the trial design and data collection, analysis, and interpretation. All authors had final responsibility for any of the above activities.

\section{Availability of data and materials}

The datasets used and/or analysed during the current study are available from the corresponding author upon reasonable request.

\section{Ethics approval and consent to participate}

The ethics committee of the UMC Utrecht approved this study (reference number: 17-933). Written informed consent will be obtained from the patient or legally authorized representative by the investigators or physician on call. Patients who are enrolled in the study are covered by the clinical trial insurance of the UMC Utrecht. The study will be conducted in accordance with the Good Clinical Practice E6(R2) guidelines and the current version of the Declaration of Helsinki.

\section{Consent for publication}

Not applicable

\section{Competing interests}

Eculizumab and research funding are provided by Alexion Pharmaceuticals under the Global Investigator-Sponsored Research Program.

Received: 10 June 2020 Accepted: 22 October 2020

Published online: 25 November 2020

\section{References}

1. Macdonald RL. Delayed neurological deterioration after subarachnoid haemorrhage. Nat Rev Neurol. 2014;10:44-58.

2. Dorhout Mees $S$, Rinkel GJ, Feigin $V L$, Algra A, van den Bergh WM, Vermeulen $\mathrm{M}$, et al. Calcium antagonists for aneurysmal subarachnoid haemorrhage. Cochrane Database Syst Rev. 2007:CD000277. https://wwwcochranelibrary-com.proxy.library.uu.nl/cdsr/doi/10.1002/14651858.CD0002 77.pub3/full.

3. Frontera JA, Provencio JJ, Sehba FA, Mclntyre TM, Nowacki AS, Gordon E, et al. The role of platelet activation and inflammation in early brain injury following subarachnoid hemorrhage. Neurocrit Care. 2017;26:48-57.

4. McMahon CJ, Hopkins S, Vail A, King AT, Smith D, Illingworth KJ, et al. Inflammation as a predictor for delayed cerebral ischemia after aneurysmal subarachnoid haemorrhage. J Neurointerv Surg. 2013;5:512-7. 
5. Savarraj J, Parsha K, Hergenroeder G, Ahn S, Chang TR, Kim DH, et al. Early brain injury associated with systemic inflammation after subarachnoid hemorrhage. Neurocrit Care. 2018;28:203-11.

6. Ahn SH, Savarraj JPJ, Parsha K, Hergenroeder GW, Chang TR, Kim DH, et al. Inflammation in delayed ischemia and functional outcomes after subarachnoid hemorrhage. J Neuroinflammation. 2019;16:213.

7. Zanier ER, Zangari R, Munthe-Fog L, Hein E, Zoerle T, Conte V, et al. Ficolin3-mediated lectin complement pathway activation in patients with subarachnoid hemorrhage. Neurology. 2014;82:126-34.

8. Mack WJ, Ducruet AF, Hickman ZL, Garrett MC, Albert EJ, Kellner CP, et al. Early plasma complement C3a levels correlate with functional outcome after aneurysmal subarachnoid hemorrhage. Neurosurgery. 2007;61:255-61.

9. Björk J, Hugli TE, Smedegård G. Microvascular effects of anaphylatoxins C3a and C5a. J Immunol. 1985;134:1115-9.

10. Ikeda K, Nagasawa K, Horiuchi T, Tsuru T, Nishizaka H, Niho Y. C5a induces tissue factor activity on endothelial cells. Thromb Haemost. 1997;77:394-8.

11. Laudes IJ, Chu JC, Sikranth S, Huber-Lang M, Guo R-F, Riedemann N, et al. Anti-C5a ameliorates coagulation/fibrinolytic protein changes in a rat model of sepsis. Am J Pathol. 2002;160:1867-75.

12. Sehba FA, Mostafa G, Friedrich V, Bederson JB. Acute microvascular platelet aggregation after subarachnoid hemorrhage. J Neurosurg. 2005;102:1094-100.

13. van Dijk BJ, Meijers JCM, Kloek AT, Knaup VL, Rinkel GJE, Morgan BP, et al. Complement $C 5$ contributes to brain injury after subarachnoid hemorrhage. Transl Stroke Res. 2019;11:678-88.

14. Pittock SJ, Lennon VA, McKeon A, Mandrekar J, Weinshenker BG, Lucchinetti CF, et al. Eculizumab in AQP4-IgG-positive relapsing neuromyelitis optica spectrum disorders: an open-label pilot study. Lancet Neurol. 2013;12:554-62.

15. Howard JF, Utsugisawa K, Benatar M, Murai H, Barohn RJ, Illa I, et al. Safety and efficacy of eculizumab in anti-acetylcholine receptor antibody-positive refractory generalised myasthenia gravis (REGAIN): a phase 3, randomised, double-blind, placebo-controlled, multicentre study. Lancet Neurol. 2017;16:976-86.

16. Vergouwen MDI, Vermeulen M, van Gijn J, Rinkel GJE, Wijdicks EF, Muizelaar $J P$, et al. Definition of delayed cerebral ischemia after aneurysmal subarachnoid hemorrhage as an outcome event in clinical trials and observational studies: proposal of a multidisciplinary research group. Stroke. 2010;41:2391-5.

17. McHugh GS, Butcher I, Steyerberg EW, Marmarou A, Lu J, Lingsma HF, et al. A simulation study evaluating approaches to the analysis of ordinal outcome data in randomized controlled trials in traumatic brain injury: results from the IMPACT Project. Clin Trials J Soc Clin Trials. 2010;7:44-57.

\section{Publisher's Note}

Springer Nature remains neutral with regard to jurisdictional claims in published maps and institutional affiliations.

Ready to submit your research? Choose BMC and benefit from:

- fast, convenient online submission

- thorough peer review by experienced researchers in your field

- rapid publication on acceptance

- support for research data, including large and complex data types

- gold Open Access which fosters wider collaboration and increased citations

- maximum visibility for your research: over $100 \mathrm{M}$ website views per year

At $\mathrm{BMC}$, research is always in progress.

Learn more biomedcentral.com/submissions 